\title{
Percutaneous needling of Morton's complex: a technical note
}

\author{
Leire Atilano ${ }^{1}$ \\ Jose Ignacio Martin ${ }^{1}$ \\ Gotzon Iglesias ${ }^{1}$ \\ Isabel Andia ${ }^{2}$ \\ 1 Department of Radiology, Interventional Echography, \\ Cruces University Hospital, Barakaldo, Spain \\ 2 Regenerative Medicine, BioCruces Health Research \\ Institute, Cruces University Hospital, Barakaldo, \\ Spain
}

Corresponding author:

Isabel Andia

Regenerative Medicine, BioCruces Health Research

Institute, Cruces University Hospital

Pza Cruces s/n

48903 Barakaldo, Spain

E-mail: iandia2010@hotmail.com

\section{Summary}

Background: the Morton's complex, i.e. fibrotic mass enfolding the medial plantar nerve, the bursa and the interdigital transverse ligament in the web space, is a common cause of pain and functional disability. Conservative and operative treatments are investigated but currently the best approach to treat the Morton's complex is unknown. Methods: we describe a non-invasive, straight forward intervention consisting on multiple percutaneous punctures, shearing the fibrotic tissue in lateromedial and anteroposterior directions. The goal is to break up fibrosis occupying the intermetatarsal space thus releasing the affected nerve from the adjacent structures, there by stimulating tissue remodelling.

Results: slow tissue remodelling occurs following sequential fibrosis cleavage through multiple needling. Needling of the intermetatarsal fibrosis is performed every eight weeks until pain resolution. Echographic changes are associated to pain reduction as measured by Visual Analogue Score (VAS). Conclusion: we present an original idea that may improve Morton's management. Upcoming prospective clinical studies have to demonstrate the symptomatic benefits and the usefulness of this novel echographic intervention.

KEY WORDS: fibrosis, intermetatarsal ligament, Morton neuroma, nerve, ultrasound, needling.

\section{Introduction}

Forefoot pain is a common complaint that can be caused by increased tissue volume due to accumulated fibrosis within the restricted intermetatarsal web space. The disorder is manifested as a sharp burning pain in the affected web space. Fibrosis development entrapping the nerve and other adjacent structures, and occupying the forefoot fat pad are at the pathophysiology of this condition which is misnamed as the Morton neuroma.

Although this terminology has been integrated in the clinical practice, appropriate nomenclature should be given based on current understanding ${ }^{1}$. At ultrasound, a hypoechoic mass of fibrosis, i.e. inelastic collagenous fibres, replaces the hyperechoic fat pad between the heads of the metatarsophalangic joints. Histological evidence points to a non-neoplastic enlargement with varying degrees of perineural fibrosis, local vascular proliferation, oedema of the endoneurium and axonal degeneration ${ }^{2}$. As the fibrotic mass enlarges, the nerve gets entrapped against the transverse intermetatarsal ligament, most often extending to the bursa. Since the disorder can involve not only the interdigital nerves but also the adjacent structures, in this article it will be referred as the Morton's complex or intermetatarsal fibrosis.

As the nerve compression becomes more severe over time, focal demyelination occurs, followed by axonal damage, and finally fibrotic scarring within the nerve. Certainly, trauma and/or mechanical stress cause connective tissue reactions that could originate from different components of the nerve connective tissue, i.e. interfascicular epineurium or perineurium. Demyelination is related to pain in $72 \%$ of feet $^{3}$. Paroxysmal neuralgia incapacitates patient rendering walking problematic and deteriorating the quality of life.

Currently, ultrasound (US) technologies offer opportunities not only for Morton diagnosis ${ }^{4}$, yet for implementing new interventions accurately and safely 5 . Conservative therapeutic options that may provide some pain relief include echoguided injections of various injectable drugs including botulinum toxin $A^{6}$, anaesthetics, or corticosteroids showing variable response rates ${ }^{7,8}$. Additionally, extracorporeal shock wave therapy ${ }^{9}$ and alcohol neurolysis ${ }^{10}$ has been reported and advocated to relieve symptoms. Conservative treatment failure is not uncommon, and then percutaneous ablation of the nerve by using radiofrequency, chemicals or cryotherapy can be used ${ }^{11,12}$. 
Eventually, surgical primary neuroma resection is performed, but not always with satisfactory results because of recurrence of symptoms due to detrimental healing. Limitations of surgery include the possibility of continued pain, sensory deficits, bursitis and difficulties with shoe wear ${ }^{13}$.

The simplest and most effective way to treat Morton's neuroma, more specifically the Morton complex, is not clear. The challenge is to break the fibrotic tissue, mechanically release the structures that form the Morton's complex (ligament, bursa and nerve); in doing so reduce pain while favouring tissue remodelling. The aim of this article is to describe a simple echoguided procedure that meets these challenges. The procedure is based on mechanical and biological concepts; the former involves break-down of the fibrosis, while releasing of entrapment. The second is based on softening the fibrotic tissue, in doing so favouring remodelling and nerve repair.

\section{Methods and patients}

The procedure meets the Ethical Standards of Muscles Ligaments Tendons Journal ${ }^{14}$.

\section{Ultrasound-guided intervention}

We perform the procedure with 4-13 $\mathrm{MHz}$ high-frequency linear probe (Esaote MyLab 70 XVG, Esaote S.p.A. Genoa, Italy). A sterile protocol is followed as for any other musculoskeletal intervention. Understanding of the relevant anatomy in normal and pathological conditions is crucial to perform the echoguided intervention successfully. Briefly, the digital nerves involved in Morton's condition originate from the medial and lateral plantar nerves, then go upwards and turn around the intermetatarsal ligament. At this point the nerve divides into medial and lateral digital branches, innervating the adjacent toes.
At echography, what we visualize is a diffuse hypoechoic mass which includes the bursa, the intermetatarsal ligament, and the interdigital nerve, but these structures are undifferentiated. We can find some vascularity in the surrounds also.

To access digital nerves and perineural fibrosis involved in the Morton-complex we perform a dorsal approach in the sagittal plane, parallel to the metatarsals in the joint area (Fig. 1A). The patient is placed in supine position with the knee flexed at an angle of $30-45^{\circ}$. Importantly, the patient's foot must be firmly fixed on the stretcher, so that the pressure applied with the probe in the metatarsal space can be stronger. Position is crucial for good visualization of the neuroma and to perform the intervention rapidly and comfortably for both patient and radiologist. The dorsal approach enters through a thinner skin thus it is more comfortable for the patient than the plantar approach $^{15}$

We widen the interdigital space between the affected toes by plantar web compression with the probe oriented in the sagittal plane. Once the neuroma is positioned for intervention, we mark the trigger point for needle entrance (Fig. 1B, 1C). Then, we puncture the skin distally at the demarcated locus, on the dorsal plane, with a 23-gauge needle (diameter $=0.60, \mathrm{~L}=25$ $\mathrm{mm}$, luerlock) angled $30-45^{\circ}$ to the plane of the foot (Fig. 1D).

The bursa and the intermetatarsal ligament are located dorsal within the Morton complex, thus they are crossed over and treated with anesthetic when approaching the nerve.

We perform about ten-twenty sequential punctures (depending on the volume of the fibrosis) in order to soften the hard fibrotic tissue, release the nerve, and induce second intention tissue repair. During needling, we deliver small volumes of $1 \%$ lidocaïne (B. Braun, Melsungen AG) to avoid pain if eventually some healthy nerve is punctured. The volume of lidocaïne depends on the volume of the fibrosis, range 5$10 \mathrm{~mL}$. Figure 2A,B shows ultrasound images before

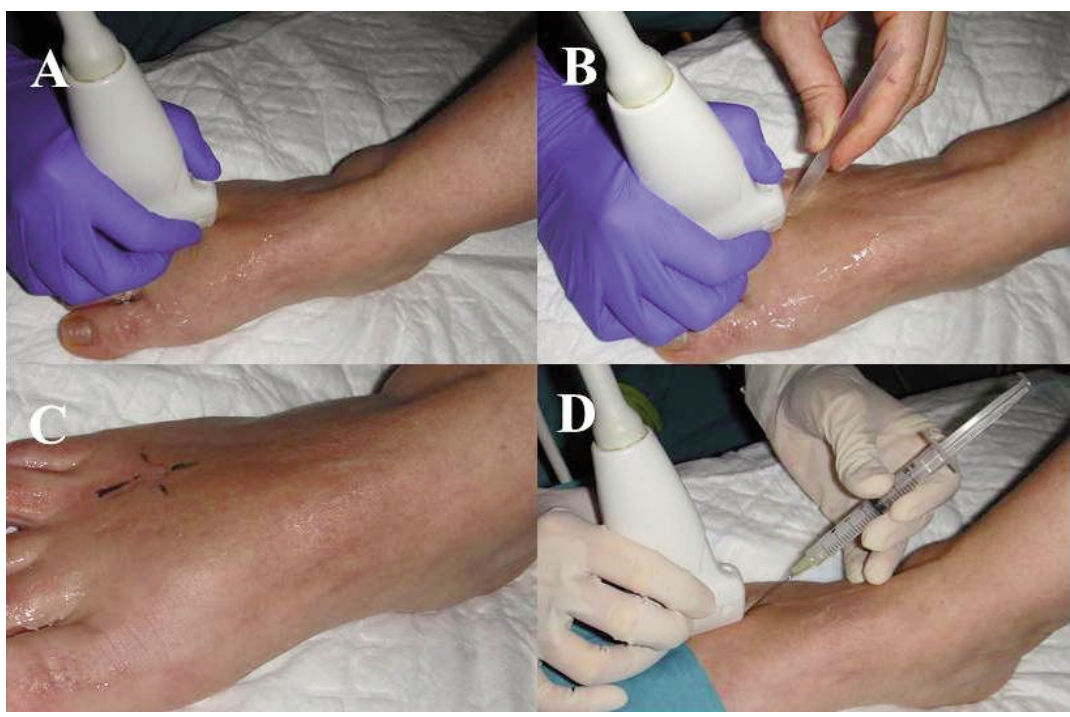

Figure 1. A) Patient lying supine with the foot fixed on the stretcher to allow widening of the intermetatarsal space. B, C) Once the neuroma is positioned for intervention, we mark the trigger point for needle entrance. D) We puncture the skin distally at the demarcated locus, with the needle angled 30 $45^{\circ}$ to the plane of the foot. 


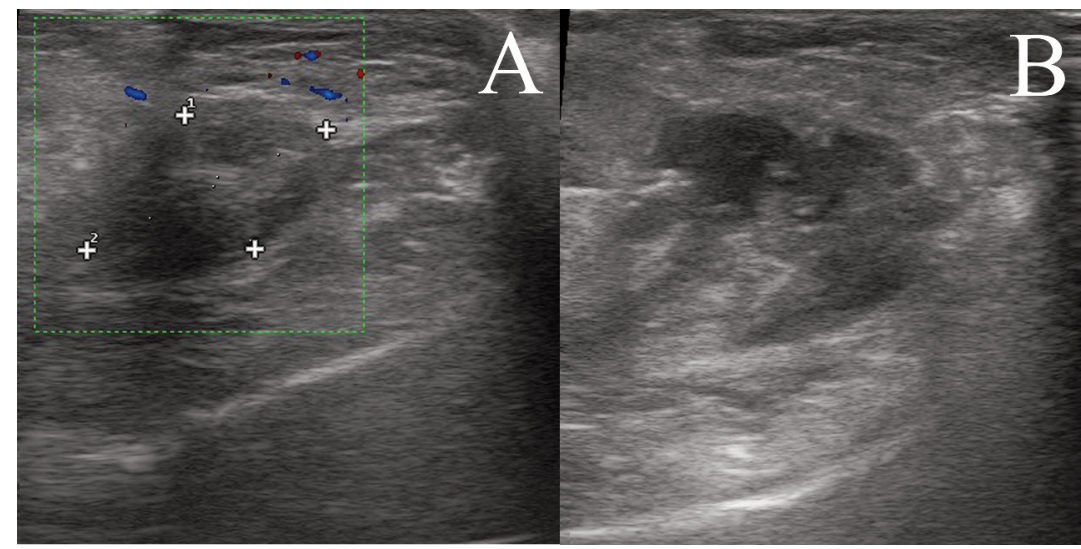

Figure 2. A) Echographic image showing the Morton's complex at baseline. B) Echographic image of the Morton's complex immediately after intervention.

and after the needling procedure. The wet needling itself provides a release of pressure by mechanically breaking up the scar fibrotic tissue that enfolds the nerve, and adheres to the ligament.

Possible risks associated to the procedure are minimal but may involve formation of a small hematoma if any vessel is accidentally reached. The patient lives the room asymptomatic. Hygienic measures regarding footwear are recommended. A follow-up visit is scheduled after 8 weeks, and if the patient refers pain, as assessed using the Visual Analogue Score (VAS), we perform a second intervention. At week 16 a third intervention can be performed if the patient experiences residual pain interfering with normal life activities.

\section{Key points}

1. Patient position and dorsal probe placement in the sagittal plane and plantar web compression to enlarge the intermetatarsal and interdigital web space.

2. Direct puncture of the Morton's complex with shearing movements while injecting anesthetic and freeing the trapped nerve. The nerve is mechanically released from surrounding fibrosis and encircled by an anesthetic ball.

3. Afterwards the nerve itself is sheared off with a small volume of anesthetics to avoid nerve herniation towards the sole of the foot.

4. The patient lives the room asymptomatic. Hygienic measures regarding footwear are recommended.

\section{Discussion}

We present novel insights into the concept and management of the Morton's complex embodied by a simple, innovative procedure based on echoguided, sequential wet needling. In doing so, we aim to address two problems, nerve decompression and tissue remodelling, by a simple echographic intervention. The shear forces generated by the repetitive needling associated to lidocaine liberate the nerve from the compressive fibrosis. Moreover, se- quential needling stimulates tissue repair and remodelling.

Revisiting the concept of Morton's complex can help in the development of novel treatments. The present view is that neuropathic pain arises from compression of interdigital nerves thus therapeutic approaches, other than palliatives, are largely focused on nerve ablation or surgical neurectomy. Among conservative procedures, corticosteroids and anaesthetic injections are palliative but have analgesic effects only in the short term. In a randomised trial Thomson et al. ${ }^{8}$ have examined the differences between US guided injections of corticosteroids and anaesthetics, or anaesthetic alone. Pain, function and global foot health was better in the corticosteroid group at three months. Another option to treat Morton is US-guided alcohol injections. In a recent report, Pasquali et al. ${ }^{16}$ have retrospectively review 508 patients, mean number of injections per neuroma was three; VAS decreased at one year and $74 \%$ of patients were satisfied with the procedure.

Alternative treatments with longer term effects such as phenol neurolysis or cryolysis are more aggressive and can have unwanted side effects. For example, cryoneurolysis has the risk of difficulties in healing of the injured area. Actually low temperatures create a hyperosmotic cell environment which constitutes a major mechanism for cell injury; thus the procedure can damage cells in the surrounding nerve tissues, and has been associated to skin necrosis. Friedman et al. ${ }^{17}$ reported clinical results retrospectively in 20 patients, 5 with painful Morton neuromas and 12 patients with stump neuromas secondary to surgery and 3 patients with peripheral neuritis. Fifteen of the twenty patients had a positive response to cryoneurolysis, of whom eleven had marked or total pain relief. Pain relief ranged from 5 months to 3 years.

Eventually, when patients do not respond to conservative therapies, surgical primary neuroma resection is performed. Bauer et al. ${ }^{13}$ compared open neurectomy versus a percutaneous procedure with deep transversal metatarsal ligament release and distal osteotomies in 52 patients. After 2 years, functional improvement persisted in most patient treated by the percutaneous procedure. Instead persistent metatarsalgia due to plantar hyperpressure or bursitis 
requiring plantar orthotics was present in $44 \%$ of patients who had undergone the surgical procedure ${ }^{13}$. Other Authors ${ }^{18}$ performed excision of Morton through a distal plantar approach, and reported fair results in $6.9 \%$ of patients and poor results in $3.8 \%$ seven years. Kasparek et al. ${ }^{3}$ reported good clinical results, although sensory deficits were very common, fair results were found in $15 \%$ of patients and poor results in $8 \%$. Complications after Morton surgery may require further regenerative surgical interventions. De Angelis et al. ${ }^{19}$ have reported the use of Platelet Rich Plasma (PRP) and hyaluronic acid to treat postoperative wound dehiscence and tendon exposures with good results.

However, surgery is not always satisfactory because of recurrence of symptoms due to the formation of stumps during postsurgical healing. Sensory deficits including numbness and concomitant foot and ankle disorders are also common after surgical treatment. Moreover, neurectomy can fail because of the persistent lateral metatarsalgia from the plantar hyperpressure often associated with Morton's but not addressed at surgery.

Overall, efforts are focused in nerve management without considering the management of fibrosis rigorously. The current point of view may lead us to ignore potentially effective details to be incorporated in the treatment. In fact, alterations in nerve physiology are evident but it is unclear if the surrounding fibrosis is the origin or the consequence.

The described needling procedure can improve substantially foot health, and it is harmless. This procedure emerged as an evolution of our standardized care protocol that consisted on corticosteroid injections. The time needed to perform the needling procedure is about $5 \mathrm{~min}$. Overall, room occupancy time is $10-20 \mathrm{~min}$, and thus it is feasible in our busy Interventional Echography Department without any additional workload. The procedure can be added to the toolbox for treating Morton's complex since it is safe, easy to perform and cost-effective. The main inconvenient is the operator learning curve, although full understanding of the foundations of the procedure facilitates implementation. The operator must be sensitive and feel when the nerve gets rid of the surrounding fibrosis, when performing the mechanical manipulation.

Further clinical research and prospective studies with long-term follow-up are guaranteed to truly assess the value of this intervention in Morton's management.

\section{Acknowledgements}

We would like to thank Mercedes Moreno and Jose Luis Perez-Anton for nursing assistance.

\section{Disclosure statement}

The Authors declare no conflicts of interest.

\section{References}

1. Larson EE, Barrett SL, Battiston B, Maloney CT Jr, Dellon AL. Accurate nomenclature for forefoot nerve entrapment: a historical perspective. J Am Podiatr Med Assoc. 2005;95(3):298306.

2. Read JW, Noakes JB, Kerr D, Crichton KJ, Slater HK, Bonar F. Morton's metatarsalgia: sonographic findings and correlated histopathology. Foot Ankle Int. 1999;20(3):153-161.

3. Kasparek M, Schneider W. Surgical treatment of Morton's neuroma: clinical results after open excision. Int Orthop. 2013; 37(9):1857-1861.

4. De Maeseneer M, Madani $\mathrm{H}$, Lenchik L, et al. Normal Anatomy and Compression Areas of Nerves of the Foot and Ankle: US and MR Imaging with Anatomic Correlation. Radiographics. 2015:150028.

5. Morgan P, Monaghan W, Richards S. A systematic review of ultrasound-guided and non-ultrasound-guided therapeutic injections to treat Morton's neuroma. J Am Podiatr Med Assoc. 2014;104(4):337-348.

6. Climent JM, Mondéjar-Gómez F, Rodríguez-Ruiz C, et al. Treatment of Morton neuroma with botulinum toxin A: a pilot study. Clin Drug Investig. 2013;33(7):497-503.

7. Hassouna $H$, Singh D, Taylor H, Johnson S. Ultrasound guided steroid injection in the treatment of interdigital neuralgia. Acta Orthop Belg. 2007;73(2):224-229.

8. Thomson CE, Beggs I, Martin DJ, et al. Methylprednisolone injections for the treatment of Morton neuroma: a patient-blinded randomized trial. J Bone Joint Surg Am. 2013;95(9):790-798, S1.

9. Fridman R, Cain JD, Weil L Jr. Extracorporeal shockwave therapy for interdigital neuroma: a randomized, placebo-controlled, double-blind trial. J Am Podiatr Med Assoc. 2009; 99(3):191-193.

10. Musson RE, Sawhney JS, Lamb L, Wilkinson A, Obaid H. UItrasound guided alcohol ablation of Morton's neuroma. Foot Ankle Int. 2012;33(3):196-201.

11. Chuter GS, Chua YP, Connell DA, Blackney MC. Ultrasoundguided radiofrequency ablation in the management of interdigital (Morton's) neuroma. Skeletal Radiol. 2013;42(1):107-111.

12. Deniz S, Purtuloglu T, Tekindur S, et al. Ultrasound-Guided Pulsed Radio Frequency Treatment in Morton's Neuroma. J Am Podiatr Med Assoc. 2015;105(4):302-306.

13. Bauer T, Gaumetou E, Klouche S, Hardy P, Maffulli N. Metatarsalgia and Morton's Disease: Comparison of Outcomes Between Open Procedure and Neurectomy Versus Percutaneous Metatarsal Osteotomies and Ligament Release With a Minimum of 2 Years of Follow-Up. J Foot Ankle Surg. 2015;54(3):373-377.

14. Padulo J, Oliva F, Frizziero A, Maffulli N. Muscles, Ligaments and Tendons Journal. Basic principles and recommendations in clinical and field science research. MLTJ. 2013;4:250-252.

15. Yap LP, McNally E. Patient's assessment of discomfort during ultrasound-guided injection of Morton's neuroma: selecting the optimal approach. J Clin Ultrasound. 2012;40(6):330-334.

16. Pasquali $C$, Vulcano $E$, Novario $R$, et al. Ultrasound-guided alcohol injection for Morton's neuroma. Foot Ankle Int. 2015; 36(1):55-59.

17. Friedman $T$, Richman D, Adler R. Sonographically guided cryoneurolysis: preliminary experience and clinical outcomes. $J$ Ultrasound Med. 2012;31(12):2025-2034.

18. De Angelis $B$, Lucarini L, Orlandi $F$, et al. Regenerative surgery of the complications with Morton's neuroma surgery: use of platelet rich plasma and hyaluronic acid. Int Wound J. 2013 10(4):372-376.

19. Nery C, Raduan F, Del Buono A, Asaumi ID, Maffulli N. Plantar approach for excision of a Morton neuroma: a long-term follow-up study. J Bone Joint Surg Am. 2012;94(7):654-658. 